\title{
Detection chain and electronic readout of the QUBIC instrument
}

\author{
Stankowiak, G., Piat, M., Battistelli, E., D'Alessandro, G., \\ de Bernardis, P., et al.
}

G. Stankowiak, M. Piat, E. Battistelli, G. D'Alessandro, P. de Bernardis, M. De Petris, M. González, L. Grandsire, J.-Ch. Hamilton, T. D. Hoang, S. Masi, S. Marnieros, A. Mennella, L. Mousset, C. O'Sullivan, D. Prêle, A. Tartari, J.-P. Thermeau, S. A. Torchinsky, F. Voisin, M. Zannoni, P. Ade, J. G. Alberro, A. Almela, G. Amico, L. H. Arnaldi, D. Auguste, J. Aumont, S. Azzoni, S. Banfi, B. Bélier, A. Baù, D. Bennett, L. Bergé, J.-Ph. Bernard, M. Bersanelli, M.-A. Bigot-Sazy, J. Bonaparte, J. Bonis, E. Bunn, D. Burke, D. Buzi, F. Cavaliere, P. Chanial, C. Chapron, R. Charlassier, A. C. Cobos Cerutti, F. Columbro, A. Coppolecchia, G. de Gasperis, M. De Leo, S. Dheilly, C. Duca, L. Dumoulin, A. Etchegoyen, A. Fasciszewski, L. P. Ferreyro, D. Fracchia, C. Franceschet, M. M. Gamboa Lerena, K. M. Ganga, B. García, M. E. García Redondo, M. Gaspard, D. Gayer, M. Gervasi, M. Giard, V. Gilles, Y. GiraudHéraud, M. Gómez Berisso, M. Gradziel, M. R. Hampel, Diego Harari, S. Henrot-Versillé, F. Incardona, E. Jules, J. Kaplan, C. Kristukat, L. Lamagna, S. Loucatos, T. Louis, B. Maffei, W. Marty, A. Mattei, A. May, M. McCulloch, L. Mele, D. Melo, L. Montier, L. M. Mundo, J. A. Murphy, J. D. Murphy, F. Nati, E. Olivieri, C. Oriol, A. Paiella, F. Pajot, A. Passerini, H. Pastoriza, A. Pelosi, C. Perbost, M. Perciballi, F. Pezzotta, F. Piacentini, L. Piccirillo, G. Pisano, M. Platino, G. Polenta, R. Puddu, D. Rambaud, P. Ringegni, G. E. Romero, E. Rasztocky, J. M. Salum, A. Schillaci, C. Scóccola, S. Scully, S. Spinelli, M. Stolpovskiy, A. D. Supanitsky, P. Timbie, M. Tomasi, G. Tucker, C. Tucker, D. Viganò, N. Vittorio, F. Wicek, M. Wright, A. Zullo, "Detection chain and electronic readout of the QUBIC instrument," Proc. SPIE 11453, Millimeter, Submillimeter, and Far-Infrared Detectors and Instrumentation for Astronomy X, 1145328 (13 December 2020); doi: 10.1117/12.2561567

Event: SPIE Astronomical Telescopes + Instrumentation, 2020, Online Only 


\section{Detection chain and Readout Electronic of the QUBIC instrument}

G. Stankowiak ${ }^{1}$, M. Piat ${ }^{1}$, E.S. Battistelli ${ }^{2,3}$, G. D'Alessandro ${ }^{2,3}$, P. de Bernardis ${ }^{2,3}$, M. De Petris ${ }^{2,3}$, M. González ${ }^{4}$, L. Grandsire ${ }^{1}$, J.-Ch. Hamilton ${ }^{1}$, T.D. Hoang ${ }^{5}$, S. Masi ${ }^{2,3}$, S. Marnieros ${ }^{6}$, A. Mennella ${ }^{7,8}$, L. Mousset ${ }^{1}$, C. O'Sullivan ${ }^{9}$, D. Prêle ${ }^{1}$, A. Tartari ${ }^{10}$, J.-P. Thermeau ${ }^{1}$, S.A. Torchinsky ${ }^{1,11}$, F. Voisin ${ }^{1}$, M. Zannoni ${ }^{12,8}$, P. Ade ${ }^{13}$, J.G. Alberro ${ }^{14}$, A. Almela ${ }^{15}$, G. Amico ${ }^{2}$, L.H. Arnaldi ${ }^{4}$, D. Auguste ${ }^{6}$, J. Aumont ${ }^{16}$, S. Azzoni ${ }^{17}$, S. Banfi ${ }^{12,8}$, B. Bélier ${ }^{18}$, A. Baù ${ }^{12,8}$, D. Bennett ${ }^{9}$, L. Bergé ${ }^{6}$, J.-Ph. Bernard ${ }^{16}$, M. Bersanelli ${ }^{7,8}$, M.-A. Bigot-Sazy ${ }^{1}$, J. Bonaparte ${ }^{19}$, J. Bonis ${ }^{6}$, E. Bunn ${ }^{20}$, D. Burke ${ }^{9}$, D. Buzi ${ }^{2}$, F. Cavaliere ${ }^{7,8}$, P. Chanial ${ }^{1}$, C. Chapron ${ }^{1}$, R. Charlassier ${ }^{1}$, A.C. Cobos Ceruttit ${ }^{15}$, F. Columbro ${ }^{2,3}$, A. Coppolecchia ${ }^{2,3}$, G. De Gasperis ${ }^{21}$, M. De Leo ${ }^{2,22}$, S. Dheilly ${ }^{1}$, C. Duca ${ }^{15}$, L. Dumoulin ${ }^{6}$, A. Etchegoyen ${ }^{15}$, A. Fasciszewski1 ${ }^{19}$, L.P. Ferreyro ${ }^{15}$, D. Fracchia ${ }^{15}$, C. Franceschet ${ }^{7,8}$, M.M. Gamboa Lerena ${ }^{23}$, K.M. Ganga ${ }^{1}$, B. García ${ }^{15}$, M.E. García Redondo ${ }^{15}$, M. Gaspard ${ }^{6}$, D. Gayer ${ }^{9}$, M. Gervasi ${ }^{12,8}$, M. Giard ${ }^{16}$, V. Gilles ${ }^{2}$, Y. Giraud-Heraud ${ }^{1}$, M. Gómez Berisso ${ }^{4}$, M. Gradziel ${ }^{9}$, M.R. Hampel ${ }^{15}$, D. Harari ${ }^{4}$, S. Henrot-Versillé ${ }^{6}$, F. Incardona ${ }^{7,8}$, E. Jules ${ }^{6}$, J. Kaplan ${ }^{1}$, C. Kristukat ${ }^{24}$, L. Lamagna ${ }^{2,3}$, S. Loucatos ${ }^{1,25}$, T. Louis ${ }^{6}$, B. Maffei ${ }^{26}$, W. Marty ${ }^{16}$, A. Mattei ${ }^{3}$, A. May ${ }^{27}$, M. McCulloch ${ }^{27}$, L. Mele ${ }^{2}$, D. Melo ${ }^{15}$, L. Montier ${ }^{16}$, L.M. Mundo ${ }^{14}$, J.A. Murphy ${ }^{9}$, J.D. Murphy ${ }^{9}$, F. Nati ${ }^{12,8}$, E. Olivieri' ${ }^{6}$, C. Oriol ${ }^{6}$, A. Paiella ${ }^{2,3}$, F. Pajot ${ }^{16}$, A. Passerini ${ }^{12,8}$, H. Pastoriza ${ }^{4}$, A. Pelosi ${ }^{3}$, C. Perbost ${ }^{1}$, M. Perciballi ${ }^{3}$, F. Pezzotta ${ }^{7,8}$, F. Piacentini ${ }^{2,3}$, L. Piccirillo ${ }^{27}$, G. Pisano ${ }^{13}$, M. Platino ${ }^{15}$, G. Polenta ${ }^{28}$, R. Puddu ${ }^{29}$, D. Rambaud ${ }^{16}$, P. Ringegni ${ }^{14}$, G.E. Romero ${ }^{30}$, E. Rasztocky ${ }^{30}$, J.M. Salum ${ }^{15}$, A. Schillaci ${ }^{31}$, C. Scóccola ${ }^{23}$, S. Scully ${ }^{9,32}$, S. Spinelli12 , M. Stolpovskiy ${ }^{1}$, A.D. Supanitsky ${ }^{15}$, P. Timbie ${ }^{33}$,

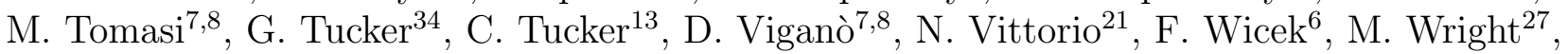
and and A. Zullo ${ }^{3}$

${ }^{1}$ Université de Paris, CNRS, Astroparticule et Cosmologie, F-75006 Paris, France

${ }^{2}$ Università di Roma - La Sapienza

${ }^{3}$ INFN Rome section

${ }^{4}$ Centro Atómico Bariloche and Instituto Balseiro (CNEA)

${ }^{5}$ University of Science and Technology of Hanoi, Vietnam Academy of Science and Technology

${ }^{6}$ Laboratoire de Physique des 2 Infinis Irène Joliot-Curie (CNRS-IN2P3, Université

Paris-Saclay)

${ }^{7}$ Universita degli studi di Milano

${ }^{8}$ INFN Milano-Bicocca

${ }^{9}$ National University of Ireland, Maynooth

${ }^{10}$ INFN - Pisa Section, 56127 Pisa, Italy

${ }^{11}$ Observatoire de Paris, F-75014 Paris, France

${ }^{12}$ Università di Milano - Bicocca

${ }^{13}$ Cardiff University

${ }^{14}$ GEMA (Universidad Nacional de La Plata)

${ }^{15}$ Instituto de Tecnologías en Detección y Astropartículas (CNEA, CONICET, UNSAM)

${ }^{16}$ Institut de Recherche en Astrophysique et Planétologie, Toulouse (CNRS-INSU)

${ }^{17}$ Department of Physics, University of Oxford

${ }^{18}$ Centre de Nanosciences et de Nanotechnologies, Orsay 
${ }^{19}$ Centro Atómico Constituyentes (CNEA)

${ }^{20}$ University of Richmond, Richmond

${ }^{21}$ Università di Roma - Tor Vergata

${ }^{22}$ University of Surrey

${ }^{23}$ Facultad de Ciencias Astronómicas y Geofísicas (Universidad Nacional de La Plata)

${ }^{24}$ Escuela de Ciencia y Tecnología (UNSAM) and Centro Atómico Constituyentes (CNEA)

${ }^{25}$ IRFU, CEA, Université Paris-Saclay, F-91191 Gif-sur-Yvette, France

${ }^{26}$ Institut d'Astrophysique Spatiale, Orsay (CNRS-INSU)

${ }^{27}$ University of Manchester

${ }^{28}$ Italian Space Agency

${ }^{29}$ Pontificia Universidad Catolica de Chile

${ }^{30}$ Instituto Argentino de Radioastronomía (CONICET, CIC)

${ }^{31}$ California Institute of Technology

${ }^{32}$ Institute of Technology, Carlow

${ }^{33}$ University of Wisconsin, Madison

${ }^{34}$ Brown University, Providence

\begin{abstract}
The Q \& U Bolometric Interferometer for Cosmology (QUBIC) Technical Demonstrator (TD) aiming to shows the feasibility of the combination of interferometry and bolometric detection. The electronic readout system is based on an array of $128 \mathrm{NbSi}$ Transition Edge Sensors cooled at 350mK readout with 128 SQUIDs at 1K controlled and amplified by an Application Specific Integrated Circuit at 40K. This readout design allows a 128:1 Time Domain Multiplexing. We report the design and the performance of the detection chain in this paper. The technological demonstrator unwent a campain of test in the lab. Evaluation of the QUBIC bolometers and readout electronics includes the measurement of I-V curves, time constant and the Noise Equivalent Power. Currently the mean Noise Equivalent Power is $\sim 2 \times 10^{-16} \mathrm{~W} / \sqrt{\mathrm{Hz}}$
\end{abstract}

Keywords: TES, SQUID, CMB, TDM

\title{
1. INTRODUCTION
}

QUBIC is a dedicated ground-based experiment, aiming to observe the Cosmic Microwave Background (CMB). It is an international collaboration including Argentina, Italy, France, Ireland, the USA, and the UK. It will focus on the polarisation of the CMB and more specifically on the B-modes. Those B-modes are the witnesses of the inflationary period of the Universe's history.

QUBIC will be deployed in Argentina, at the Alto Chorillo mountain site (height: $4869 \mathrm{~m}$ ). The Technical Demonstrator (TD) is the first configuration of QUBIC. It is composed of a quarter of the focal plane (256 TES) on the $150 \mathrm{Ghz}$ band, an array of 64 horns and switches, and a smaller optical combiner. The other configuration is the Final Instrument (FI), it is composed of the same cryostat as the TD, and will be upgraded directly on the site in a near future. The TD is used to demonstrate the feasibility of the bolometric interferometry. It has been tested at APC laboratory since 2018 and still under a test campaign. (more information on the QUBIC experiment $^{1}$

This paper describes the overall design of the detection chain and the results of the characterization phase.

This paper is organized as follows; an overview of the QUBIC readout system to explain its design. Then the characterization of the different parts of the Detection Chain. To conclude, the presentation of the milestones that QUBIC reached during the underwent test campaign.

Further author information: (Send correspondence to Guillaume Stankowiak)

Guillaume Stankowiak: E-mail: guillaume.stankowiak@apc.in2p3.fr 


\section{QUBIC READOUT SYSTEM}

QUBIC readout system is described in Figure 1. It is made of 4 different parts: Transition Edge Sensor (TES), Superconducting QUantum Interferences Devices (SQUID), Application Specific Integrated Circuit (ASIC), and Field Programmable Gate Array (FPGA) which are described in the following section. Each part is working at a different temperature, with a quarter of the focal plane composed of 256 pixels TES working at $320 \mathrm{mK}$ and cooled down with a $3 \mathrm{He}$ fridge.

For each quarter of the focal plane, two blocks composed of 128 SQUID operating at $1 \mathrm{~K}$ and cooled down by a 4He fridge, are used in a 128:1 Time Domain Multiplexing (TDM) system.

For each block of 128 SQUIDs, there is an ASIC used as controller and amplifier, cooled down at 40K by two Pulse Tubes.

For each ASIC, a warm readout device: FPGA is used to ensure the control and acquisition by the DAQ computer.
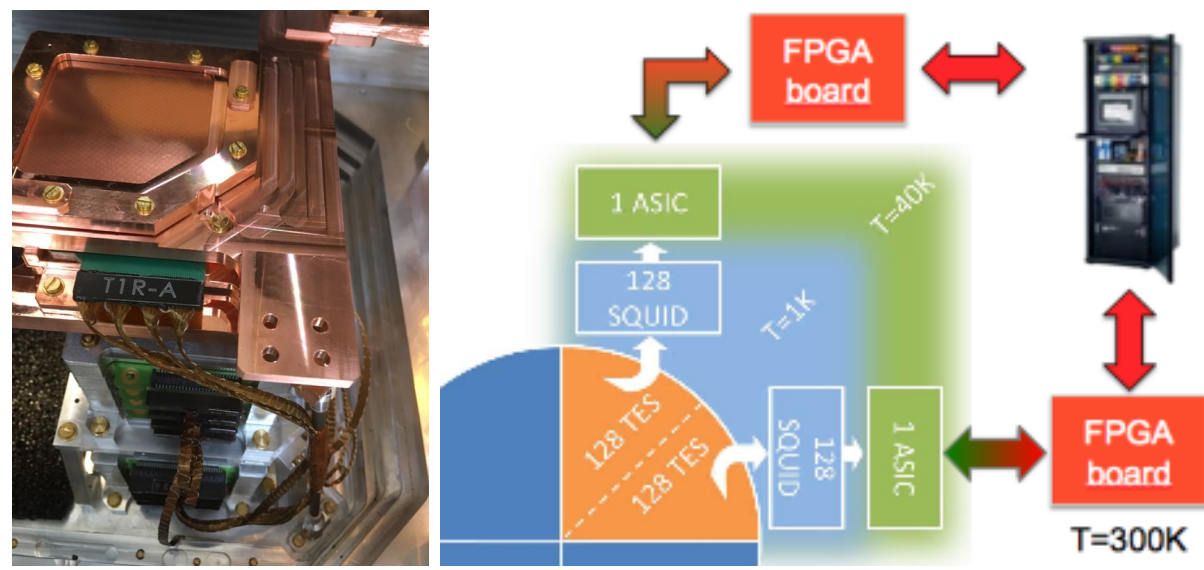

Figure 1. Left, Pictures of the TESs (top) and SQUIDs (boxes under the copper plate) in the laboratory. Right, schematic of the detection chain (colorful figure available online

\subsection{Transition Edge Sensor (TES)}

The detectors are Transition Edge Sensors (TES) made with a $\mathrm{Nb}_{\mathrm{x}} \mathrm{Si}_{1-\mathrm{x}}$ amorphous thin film ( $\mathrm{x} \approx 0.15$ in our case). Its transition temperature $\mathrm{T}_{\mathrm{c}}$ (Fig. 2) can be adapted by changing the composition $x$ of the compound. The array currently used (reference P87) has a critical temperature of about $410 \mathrm{mK}$. The normal state resistance $\mathrm{R}_{\mathrm{n}}$ is adjusted to about $1 \Omega$ with interleaved electrods for optimum performances. To adapt to the optics, the pixels have $3 \mathrm{~mm}$ spacing while the membranes structure is $2.7 \mathrm{~mm}$ wide without any sensitivity to polarization. The low thermal coupling between the TES and the cryostat is obtained using $500 \mathrm{~nm}$ thin SiN suspended membranes, which exhibit thermal conductivities in the range $50-500 \mathrm{pW} / \mathrm{K}$ depending on the precise pixel geometry and temperature. The Noise Equivalent Power (NEP) is of the order of $5.10^{-17} \mathrm{~W} / \sqrt{\mathrm{Hz}}$ at $150 \mathrm{GHz}$ with a natural time constant of about $100 \mathrm{~ms}\left[{ }^{2}\right]$. Light absorption is achieved using a Palladium metallic grid placed in a quarter wave cavity in order to optimize the absorption efficiency. The backshort distance of $400 \mu \mathrm{m}$ has been chosen after electro-magnetic simulations in order to have absorption higher than $94 \%$ at both 150 and $220 \mathrm{GHz}$.

\subsection{SQUIDs}

The QUBIC detection chain second stage is composed of the Superconducting QUantum Interference Devices (SQUIDs) maintained at a temperature of about $1 \mathrm{~K}$ by an ${ }^{4} \mathrm{He}$ fridge. Each TES is in series with the input inductance $L_{\mathrm{in}}$ of the SQUID and is voltage biased with a $10 \mathrm{~m} \Omega$ resistor in parallel as shown in Figure 3 . The input inductance of the SQUID converts the TES current into a magnetic flux $\Phi_{\text {in }}$ that is converted in an output voltage by the SQUID. The later is therefore a trans-impedance amplifier with a gain of the order of $100 \mathrm{~V} / \mathrm{A}$. In addition of being a cryogenic amplifier, SQUIDs also enable the multiplexing because of their large bandwidth. 

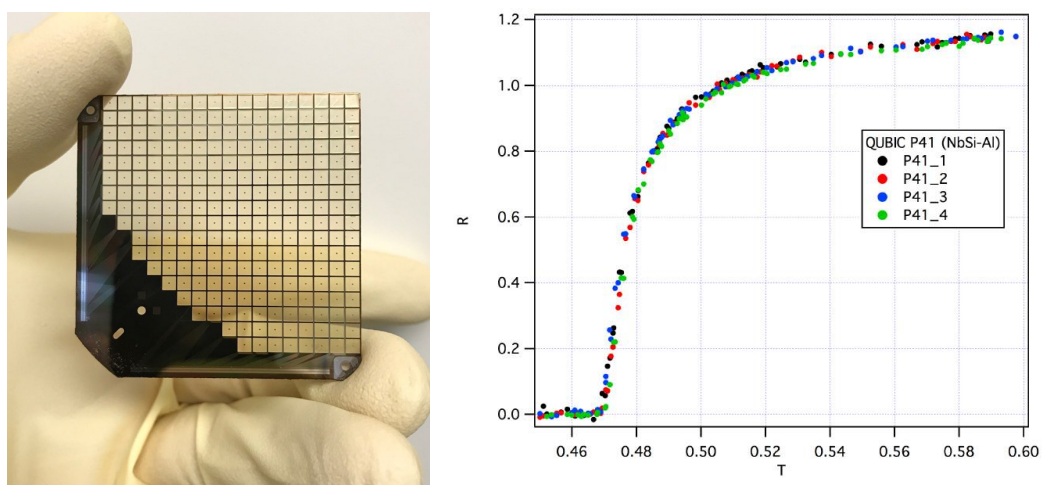

Figure 2. Left, Distribution of the Tc on the focale plane.Right, histogram of the Tc

As shown in Figure 3, the SQUID multiplexer is composed of 4 columns of 32 SQUIDs AC-biased with capacitors in order to reduce power dissipation and noise.

The SQUIDs used in QUBIC shown in Figure 3 Right have a dual-washer gradiometric layouts. They are based on a slightly modified SQ600S commercial design provided by StarCryoelectronics* in order to reduce the area of each dies. Visual inspections and room temperature tests with a probe-station are used to select the SQUIDs before integration on a specific Printed Circuit Board (PCB). One SQUID PCB is composed of 32 SQUIDs and is integrated in an aluminium box. The architecture therefore uses 4 of this PCB boxes to readout 128 pixels. As shown in Fig. 3 Left, a stack of 8 SQUID boxes is installed at 1K below the TESs in the cryo-mechanical structure, surrounded with a Cryophy ${ }^{\dagger}$ magnetic shield.
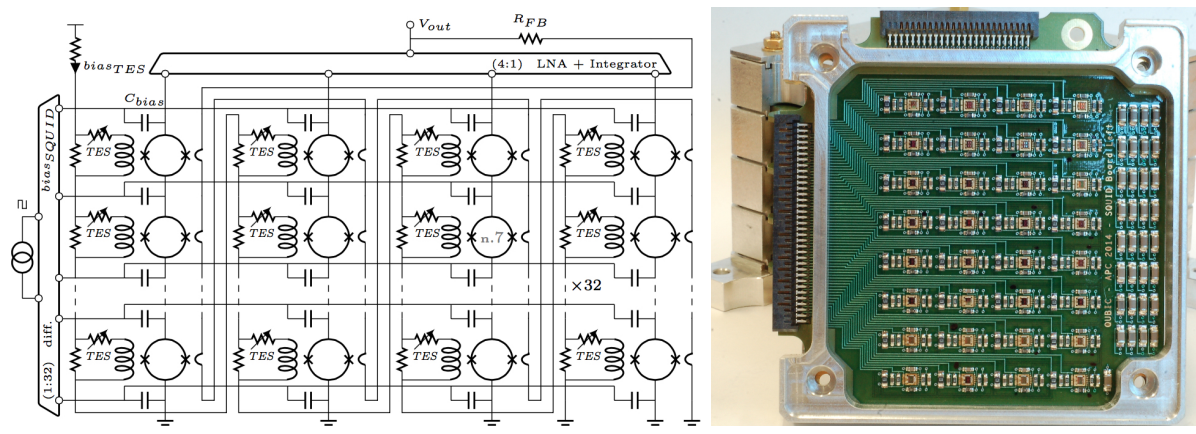

Figure 3. Left: Topology of the 128 to 1 multiplexer sub-system ( $4 \times 32$ SQUIDs +1 ASIC). Right: Integration of 32 SQUIDs (1 column) with bias capacitors and filter devices. (Color figure online.)

\subsection{ASIC}

The ASIC is designed in full-custom using CADENCE CAD tools. The used technology is a standard $0.35 \mu$ BiCMOS SiGe from Austria MicroSystem (AMS). This technology consists of p-substrate, 4-metal and $3.3 \mathrm{~V}$ process. It includes standards complementary MOS transistors and high speed vertical SiGe NPN Heterojunction Bipolar Transistors (HBT). Bipolar transistors are preferentially used for the design of analog parts because of their good performances at cryogenic temperature $\left[{ }^{3}\right]$. The design of the ASIC is based on pre-experimental characterizations results, and its performance at cryogenic temperature is extrapolated from simulation results obtained at room temperature, using CAD tools. Each ASIC board for QUBIC (shown on Fig. 4) has a power dissipation of typically $16 \mathrm{~mW}$ and is placed on the 40K stage. The ASIC integrates all parts needed to achieve the readout, the multiplexing and the control of an array of up to 128 TESs/SQUIDs. It includes a differential

\footnotetext{
*starcryo.com

${ }^{\dagger}$ www.aperam.com
} 


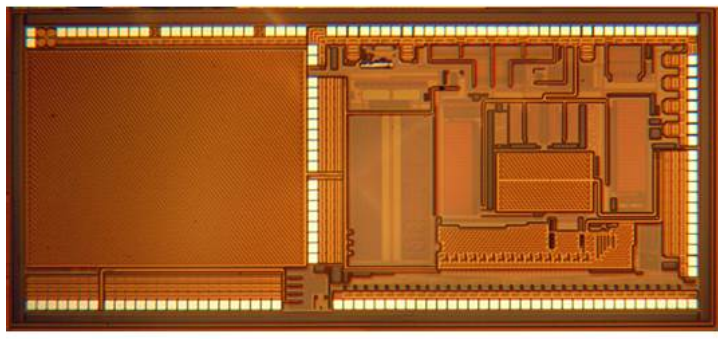

(a)

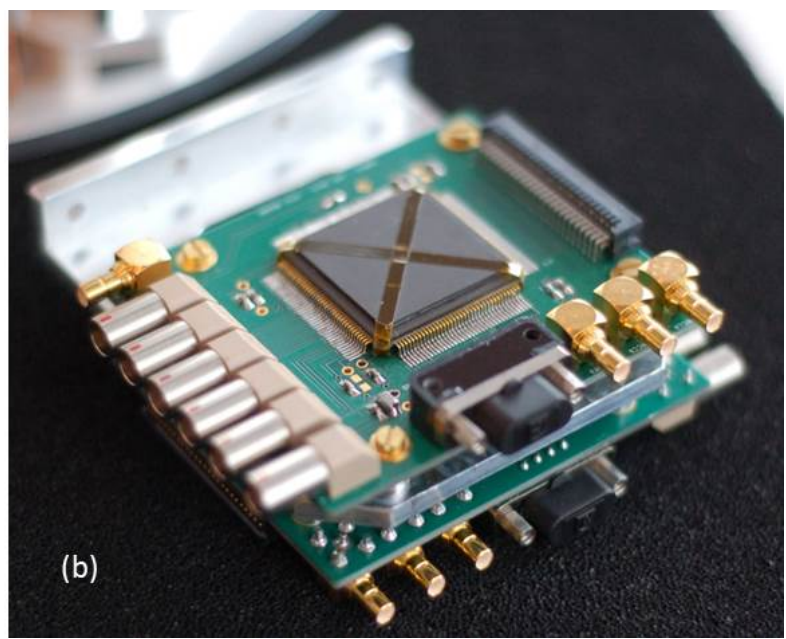

Figure 4. Left: Microphotography of cryogenic ASIC designed to readout $4 \times 32$ TES/SQUID pixels. Right: ASIC module assembly used for QUBIC experiment. (Color figure online.)

switching current source to address sequentially 32 lines of SQUIDs, achieving a first level of multiplexing of 32:1. In this configuration, the SQUID are AC biased through capacitors which allows a good isolation (low crosstalk between SQUID columns) and no power dissipation. A cryogenic SiGe low noise amplifier $\left(e_{n}=0.3 \mathrm{nV} / \sqrt{\mathrm{Hz}}\right.$, gain $=70$, bandwidth of about $6 \mathrm{MHz}$ in simuations) with 4 multiplexed inputs, performs a second multiplexing stage between each column.

This cryogenic ASIC includes also the digital synchronization circuit of the overall multiplexing switching (AC current sources and multiplexed low noise amplifier). A serial protocol allows to focus on sub-array as well as to adjust the amplifiers and current sources with a reduced number of control wires. As the digital side takes a large part, we have developed a full custom CMOS digital library dedicated to cryogenic application and ionizing environments (rad-hard full custom digital library) [3].

\subsection{Warm electronics and acquisition software}

The warm electronics is based on FPGA boards called NetQuiC, one for each ASIC. These boards are connected to the acquisition computer via a network switch. Each NetQuiC board is based on a differential amplifier (gain=100, bandwidth limited to $1 \mathrm{MHz}$ with a second order low-pass filter), a 2MHz 16 bits ADC, 716 bits DACs and a Xilinx Spartan 6 FPGA (XEM6010 board from Opal Kelly). The FPGA firmware programmed in VHDL takes in charge the following tasks: ASICs control, management of the TCP/IP connection with the acquisition computer, acquisition of scientific signal with the ADC, bias generation and digital Flux Locked Loop (FLL) control. The acquisition software called QUBIC Studio is the single interface to deal with the readout, the control command software and the data storage. Its core is the generic tool called "dispatcher" developed at IRAP that is a real-time-oriented acquisition system widely used on various experiments such as Solar Orbiter, SVOM/ECLAIRS and PILOT.

\section{CHARACTERIZATION OF THE DETECTION CHAIN}

The QUBIC TD test campaign has been done first in blind configuration (the $40 \mathrm{~K}$ filters being closed) in December 2018 and afterward with all filters open from mid-January to end of October 2020. The TESs were responsive enough to allow us to make a first complete optical characterization of the instrument (see $\left[{ }^{4}\right]$ for further details). We report here the characterizations made on the detection chain.

\subsection{ASICs}

Both ASICs 1 and 2 have been functionally tested and characterized at low temperature during the TD test campaigns. Low noise multiplexed amplifier characterizations have been investigated using a spectrum analyzer. 
Figure 5). A white noise level of $0.3 \mathrm{nV} / \sqrt{\mathrm{Hz}}$ and a knee frequency of about $400 \mathrm{~Hz}$ were measured at $70 \mathrm{~K}$ with a differential voltage gain of 70 (measured in a specific cryogenic test bench).
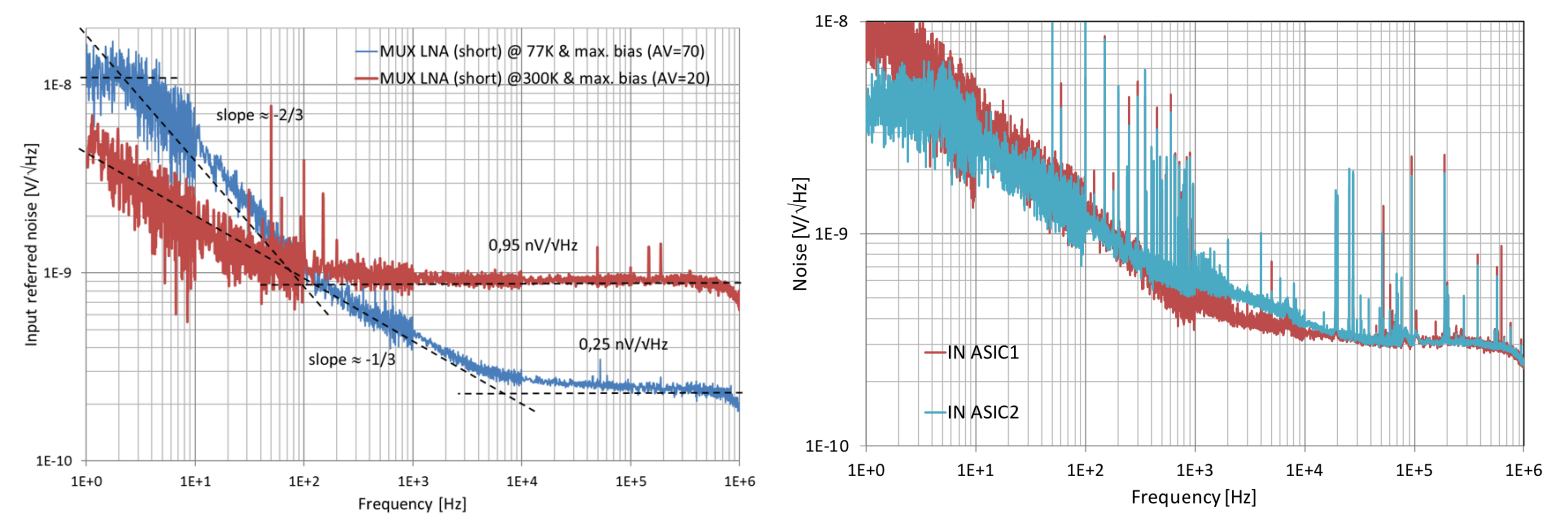

Figure 5. Multiplexed LNA (low noise amplification) equivalent input noise voltage measurement at $77 \mathrm{~K}$

\subsection{SQUIDs}

\subsubsection{Selection at warm temperature}

Before installation in QUBIC, the manufactured SQUIDs underwent a visual inspection in a clean room in order to detect and remove the ones exhibiting evidence of defects during fabrication or storage. We further proceed in the measurement of 4 resistance values at room temperature: heater, SQUID washer, feedback inductance and input inductance. An histogram of the measured values is plotted in Figure 6. SQUIDs with all parameters
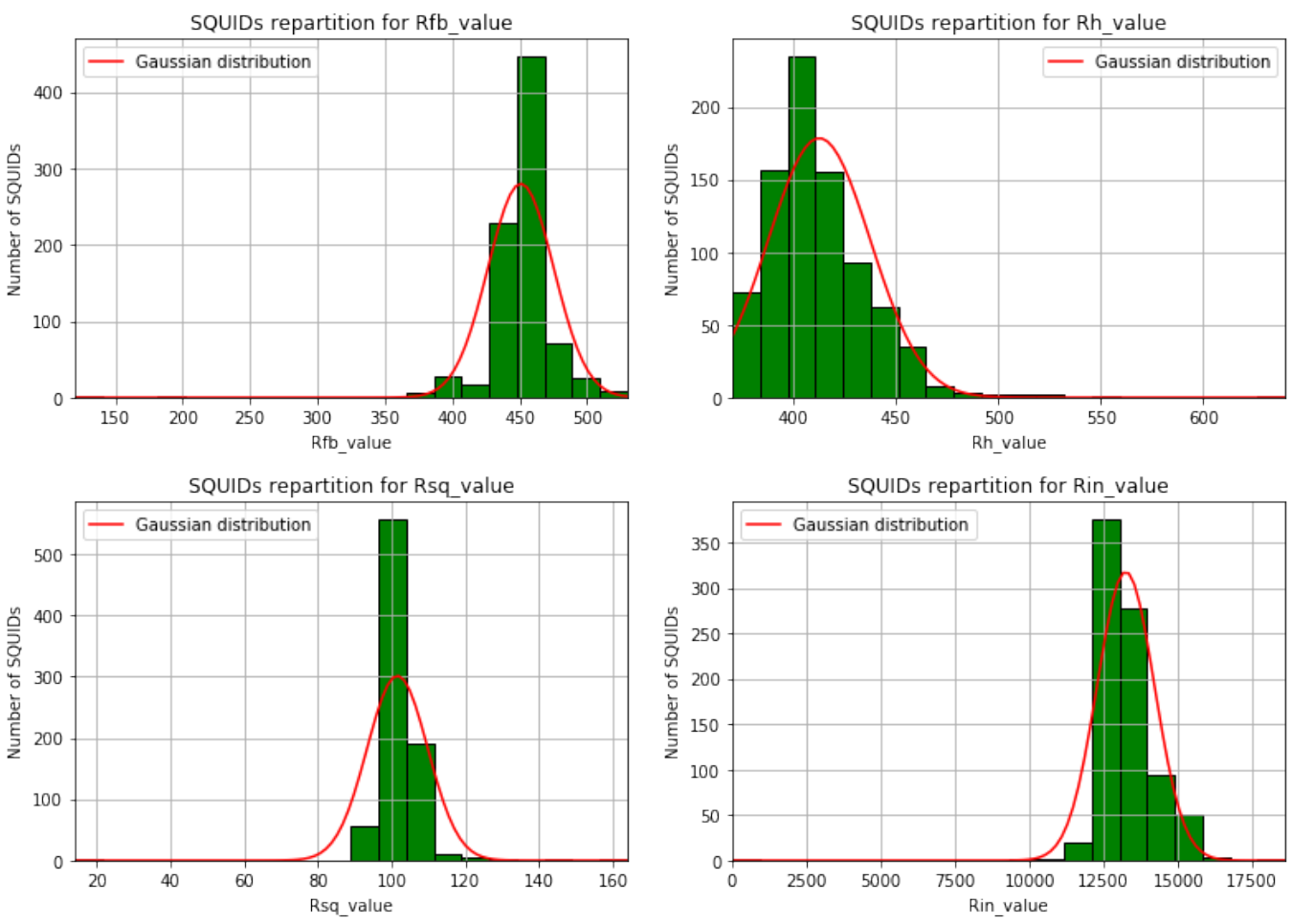

Figure 6. Plot of the value of the SQUIDs (Ohms). From the left to the right : (a) feedback value, (b) heater value, (c) SQUID value, (d) inductance value 
within $2 \sigma$ of the mean values are selected for installation in QUBIC. SQUIDs that are between $2 \sigma$ and $3 \sigma$ for one or more measurements are held aside as a possible option in case there are not enough SQUIDs passing the first criteria. All SQUIDs with any parameter larger than $3 \sigma$ from the mean are rejected. A further selection process is performed based on the leakage resistance between SQUID washer and the input inductance. Leakage measured at cryogenic temperature is typically a few M $\Omega$ between a full stack 32 SQUID and the 32 input inductances. This level of leakage does not significantly degrades operation of the SQUIDs. The pass/fail level for leakage to the input inductance was therefore set at $2 \mathrm{M} \Omega$, with the majority of leakage values measured closer to $20 \mathrm{M} \Omega$. SQUIDs with leakage to the input inductance less than $2 \mathrm{M} \Omega$ were rejected. For the leakage between the SQUID washer and the feedback, it must be an open circuit (resistance $>40 \mathrm{M} \Omega$ ), otherwise the SQUID is rejected. We typically obtained a yield of about $80 \%$ for a package of $16 \times 16$ SQUIDs.

\subsubsection{Tests at Cryogenic Temperature}

The characterization of the SQUIDs is performed at the beginng of the calibration phase. Its mains goal is to define the optimal SQUID bias current to be used during observations.

The principle of the procedure is the following: an input sinusoidal signal of $1 \mathrm{~V}$ peak-to-peak amplitude is injected on the feedback inductance through the feedback resistor $R_{\mathrm{fb}}$ and the bias current of the SQUIDs is increased step by step. For each value of the input current $I_{\text {sq }}$, the response of the SQUID is therefore measured as shown in Figure 7.
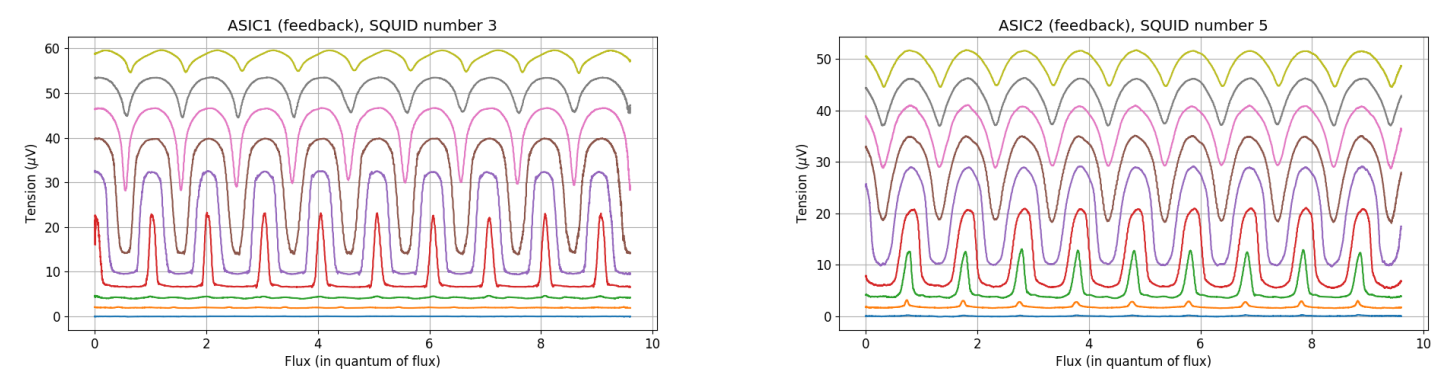

Figure 7. Flux to voltage SQUID transfer function for current biasing on ASIC 1 (Left) and on ASIC 2 (Right). The plots show the response signal $\left(V_{\mathrm{sq}}\right)$ as a function of the quantum flux going through the SQUID. There are 9 curves corresponding to increasing bias current $\left(I_{\mathrm{sq}}\right)$ (colorfull graph available online).

As the SQUID current $I_{\mathrm{sq}}$ increases, the amplitude of the response of the SQUID also increases until it reach a maximum and then it decreases. The optimum $I_{\mathrm{sq}}$ corresponds to the maximum amplitude of the SQUID response. Since the same $I_{\mathrm{sq}}$ must be supplied to all SQUID per ASIC, it is necessary to select a single bias index for all the SQUIDs for each ASIC. While it seems natural to choose the SQUID current bias corresponding to the majority of the SQUIDs, in reality it does not maximize the number of operational SQUIDs. A SQUID is considered operational if its response is greater than $10 \mu \mathrm{V}$. The SQUID current is therefore chosen to maximize the number of operational SQUIDs. Figure 8 shows the histograms of the SQUID response for three $I_{\mathrm{sq}}$ bias current for both of the ASICs.

The histograms of Figure 8 show that index 10 is the best bias index for ASIC 1 for which $93 \%$ of the SQUIDs are operational. For ASIC 2, the histograms give index 11 as the best bias index with $91 \%$ operational SQUIDs.

The yield of SQUIDs for the QUBIC Technological Demonstrator is $93 \%$ for the 128 SQUIDs connected to ASIC 1, and $89 \%$ for the 128 SQUIDs connected to ASIC 2. This corresponds to 119 operational SQUIDs for ASIC 1 and 114 operational SQUIDs for ASIC 2. The total yield is therefore 91\%. The optimum bias current is $28.06 \mu \mathrm{A}$ for ASIC 1 (bias index 10) and $30.61 \mu \mathrm{A}$ for ASIC 2 (bias index 11).

\subsection{TESs}

\subsubsection{TES parameters}

The TESs have been characterized both in the blind and open configuration. In the closed configuration, it demonstrate the operation of the TES in ETF mode in the lowest bias voltage as expected. The matrix show a 

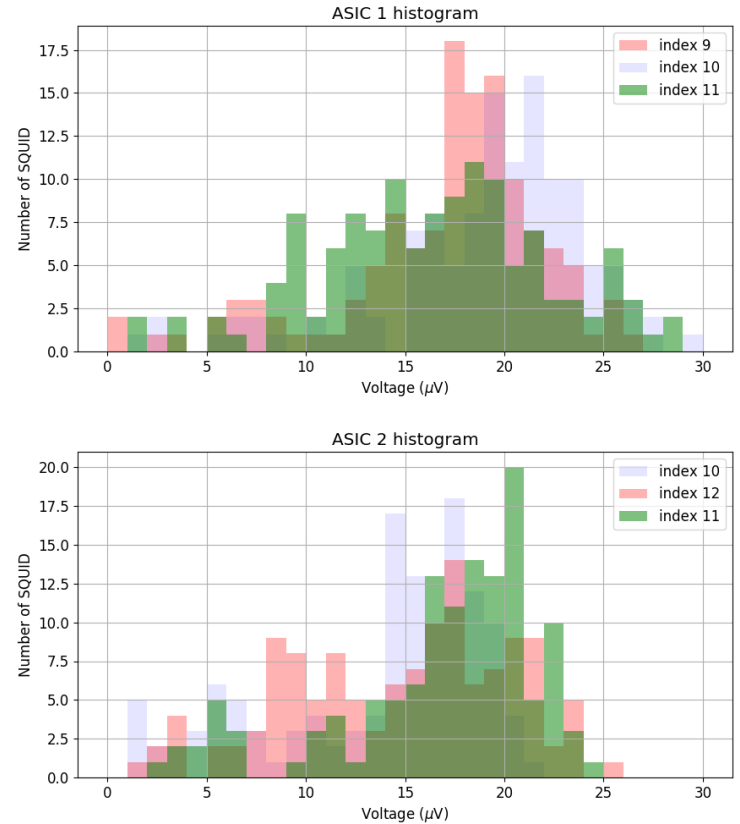

Figure 8. Histograms of the number of operational SQUIDs for each $I_{\mathrm{sq}}$ index for both ASICs.(colorfull histogram available online)

high homogeneity (Fig 9) of the TES parameter which are empiricaly find with the following equations :

$$
P_{\text {bias }}=K\left(T_{c}^{n}-T_{\text {bath }}^{n}\right),
$$

where $T_{\text {bath }}$ is the bath temperature, $K$ a constant, and $n$ the index of the power law. The $n$ value is strictly related to the signal carriers and generally has a value around 3.3. The TES thermal conductance is estimated from eq. (1) as:

$$
G=\frac{d P_{b i a s}}{d T_{c}}=K n T_{c}^{n-1}
$$

The dynamic thermal conductance 10 is measured at about $250 \mathrm{pW} / \mathrm{K}$ leading to a NEP of $5-6.10^{-17} \mathrm{~W} / \sqrt{\mathrm{Hz}}$ at $350 \mathrm{mK}$. Moreover, an overall yield of about $79 \%$ is obtained on this array (including SQUIDs yield and bad pixels). In the open configuration, the fact that the $\mathrm{R}(\mathrm{T})$ curve still has a slope in the higher part as seen in Fig. 2 allow us to get a high response to do a full optical characterization with a calibration source $\left[{ }^{4}\right]$ without the nominal sensitivity.

\subsubsection{TES Time Constante}

Data were taken on 2020 October 16 with the following configuration:

- Source modulation $0.6 \mathrm{~Hz}$, square signal, amplitude 0.5 V, offset 1V, Duty Cycle $33 \%$

- no eccosorb neither on the window nor on the CalSrc horn

- For each of the bias voltages in $[1,2,3,4,5]$ Volts we have taken around 10 minutes data

We do a very mild filtering on the data as the signal is bright and we do not want the filtering to alter the time constants. We then fold the data for each TES into one period of the calibration source. The filtering and the resulting folded signal is shown in Figure 11 for a TES picked randomly. The signal peaks can be easily seen. 


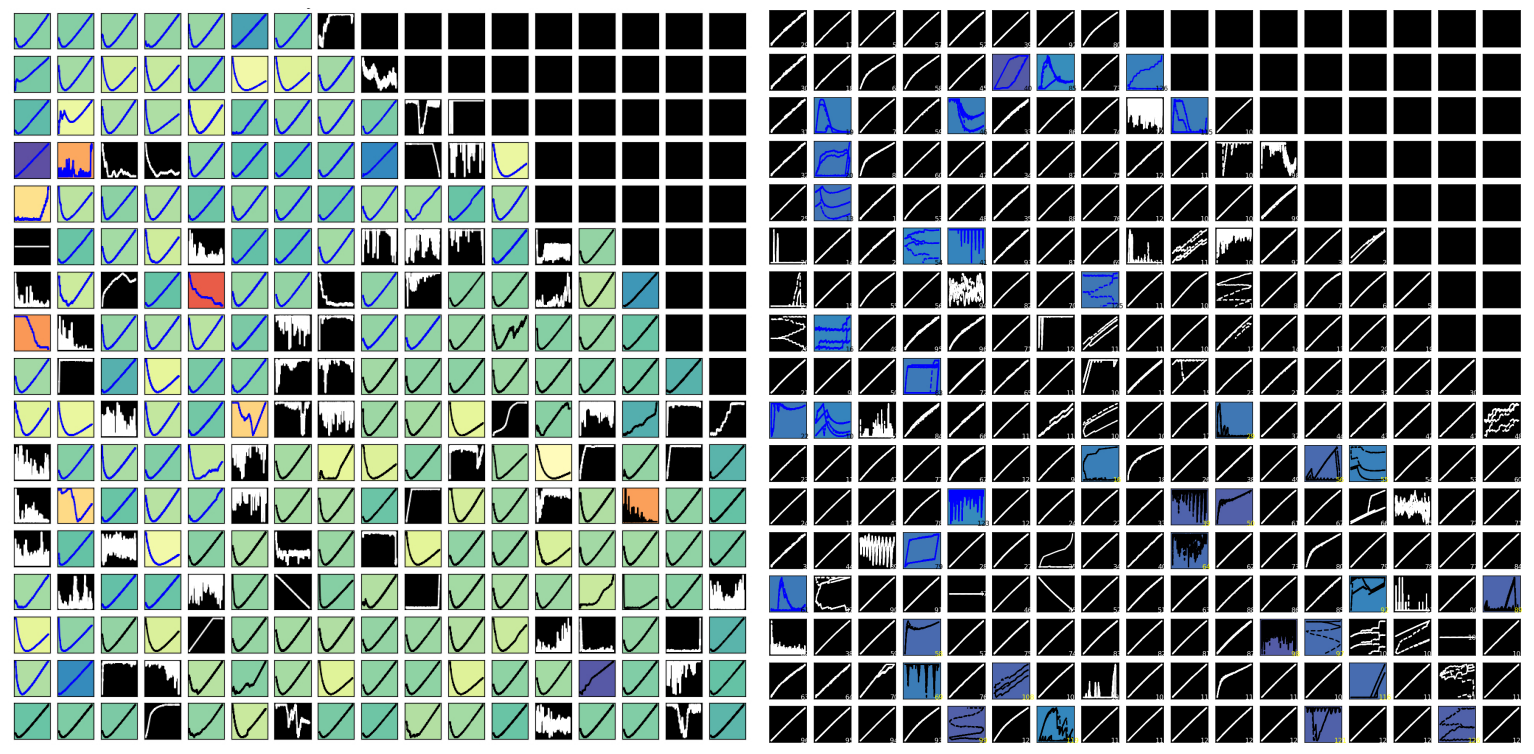

Figure 9. Left: I-V curves of the QUBIC P87 TES array measured in the blind configuration at $348 \mathrm{mK}$ down to about $60 \%$ of the normal resistance. The color code indicate the turn-over bias voltage. Right: same array measured in open configuration at $382 \mathrm{mK}$. (Color figure available online)
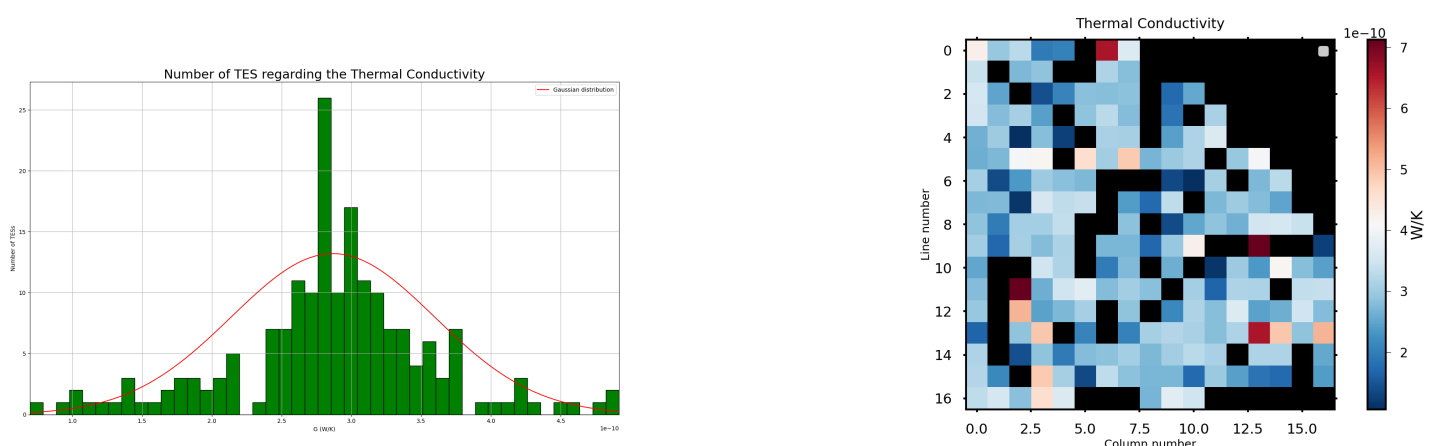

Figure 10. Left: histogramm of Thermal Conductivity Right: mapping of Thermal Conductivity .(colorfull graph available online) 

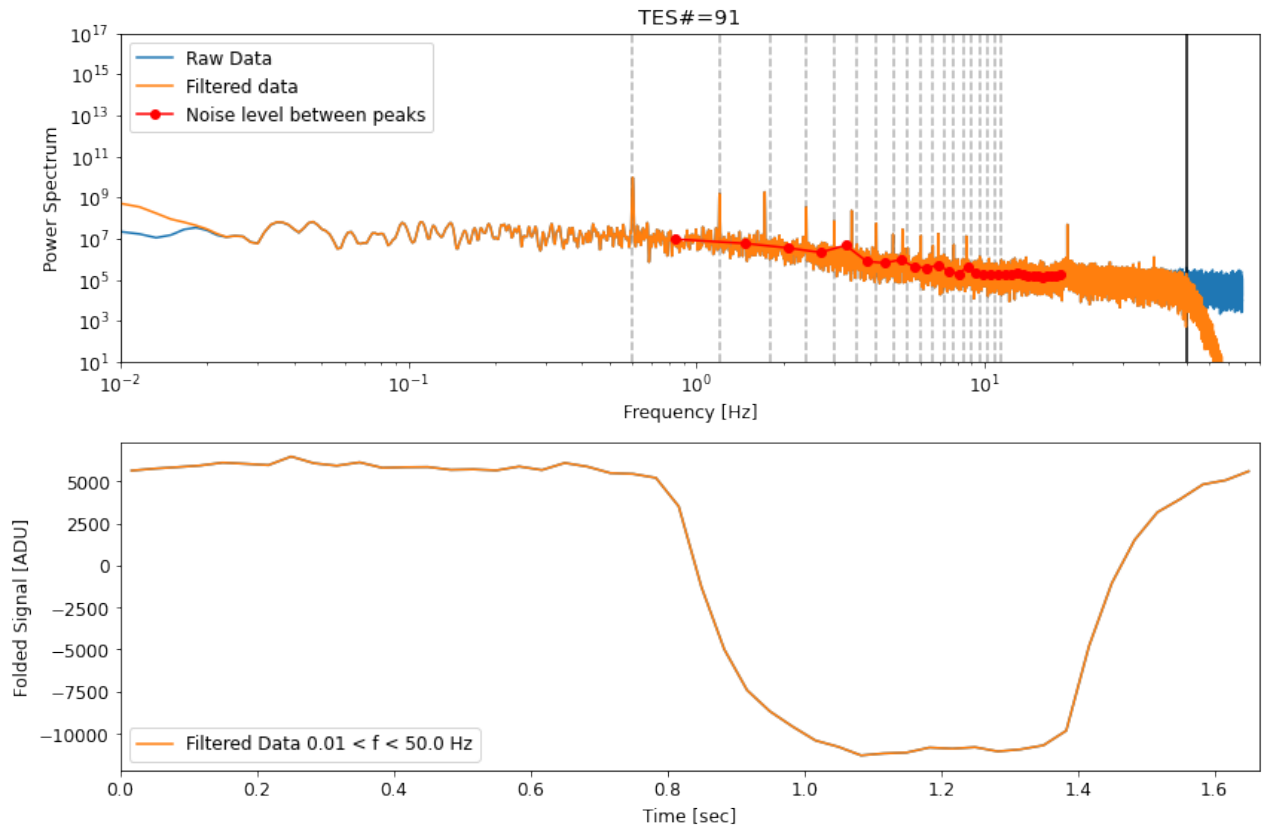

Figure 11. Folded signal for TES\#91. upper: The power spectrum. lower: The folded signal for whole time.(Color figure available online) series.

Figure 12 shows the normalized (removed average and divided by RMS) folded data for each TES in black, the median is shown in red. The derivative is shown in blue and helps finding the first guess for the start-time of the calibration source shown as a red dot. Note that no attempt is made at this stage to remove bad TES, this is why there are obvious bad signals in the plot below.

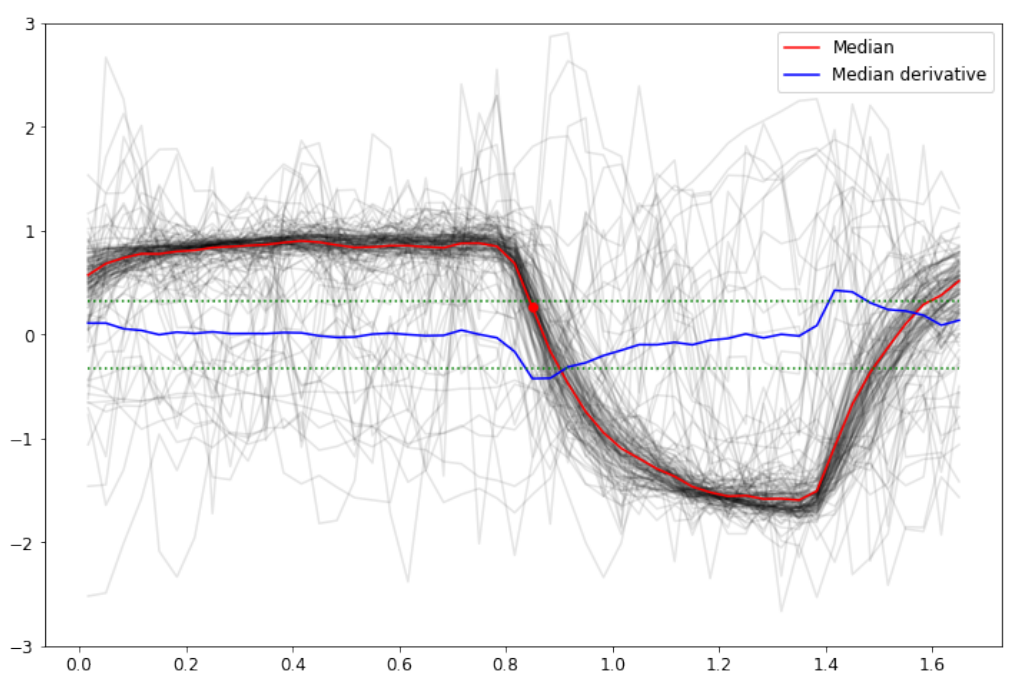

Figure 12. Normalized folded data for each TES in black, the median is shown in blue. No filtering was done to remove curves for bad TES.

We fit each TES folded signal (not normalized - meaning with its proper amplitude) with a model for the calibration source signal including time constants.

For each TES, we plot the time constant as a function of the bias voltage $V_{T E S}$ (Fig 13). On thoses plots 
we saw an acceleration of the time response atlower bias voltage which is expected from the Electro-Thermal Feedback behavior.
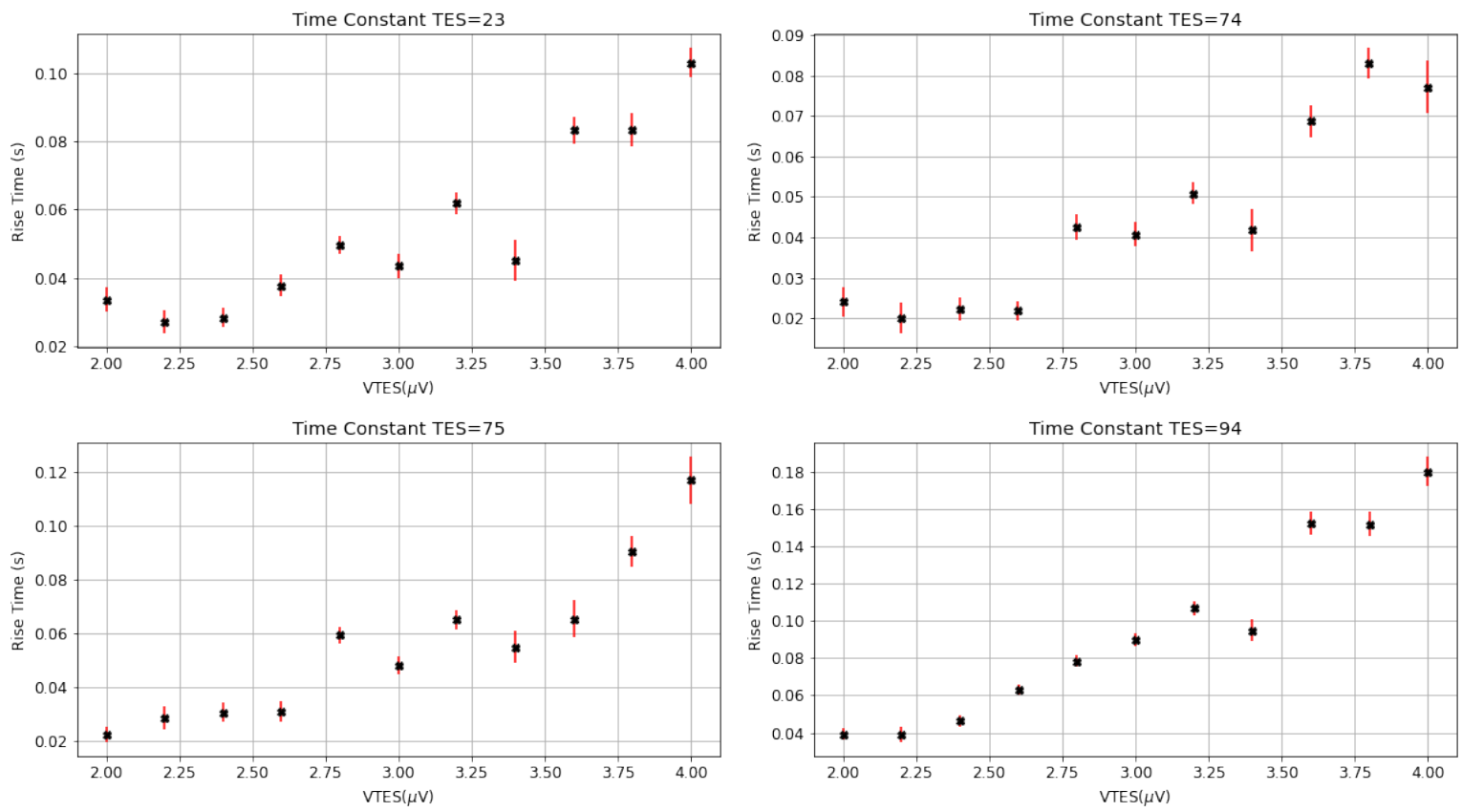

Figure 13. Time constant of TES on P87 matrix in QUBIC

An important effect shows on plots 14 is the diminution of the time constant. As $V_{T E S}$ decrease, we have an improvement of the time constant on the focal plane, from about $100 \mathrm{~ms}$ to $20 \mathrm{~ms}$ regarding the higher number of the TES on the time constant value. This means an improvement of the signal detection and so on the SNR

\section{TES NOISE PERFORMANCE}

As a very precise instrument, QUBIC is very sensitive to noise. A test of sensitivity to pulse tube (PT) microphonics was carried out by stopping the two units for a few minutes. An example timeline and associated time-frequency analysis is shown in Figure 15. The low frequency part of the noise level is reduced when both PTs are off, corresponding to the detector bandwidth. The induced parasitic signal is therefore thermal on the detector.

By the study of the current noise level, which can be converted in NEP assuming the TES are in strong ElectroThermal Feedback mode. In this case, the TES responsivity $\Re[A / W]$ is given by the inverse of the TES voltage, $\Re=\frac{1}{V_{T E S}}$. The TES voltage is obtained from the bias voltage assuming the TES resistance is higher than the shunt resistance: $V_{T E S}=V_{\text {bias }} \times 10^{-6}$.

Figure 16 shows the distribution in NEP for each cases: PTs on or off. It appears that the median NEP for the 2 cases are off from specification by a factor 7 and 2.5 respectively. Some optimizations could be further done in terms of biasing the detectors but we are clearly dominated by the PT microphonics.

As we are dominated by PT microphonics, we investigate the origin of these perturbations. We assume that the pulse tube vibrations are exciting mechanical resonance on the TES support structure but also on the TES themselves. This mechanical resonance further dissipates heats on different parts of the system.

This assumption is based on the following hints :

- In Figure 15 after the PTs are switched off, we see a small increase in the TES current which is due to a small cooling of the detector, before heating up due to background increase. 

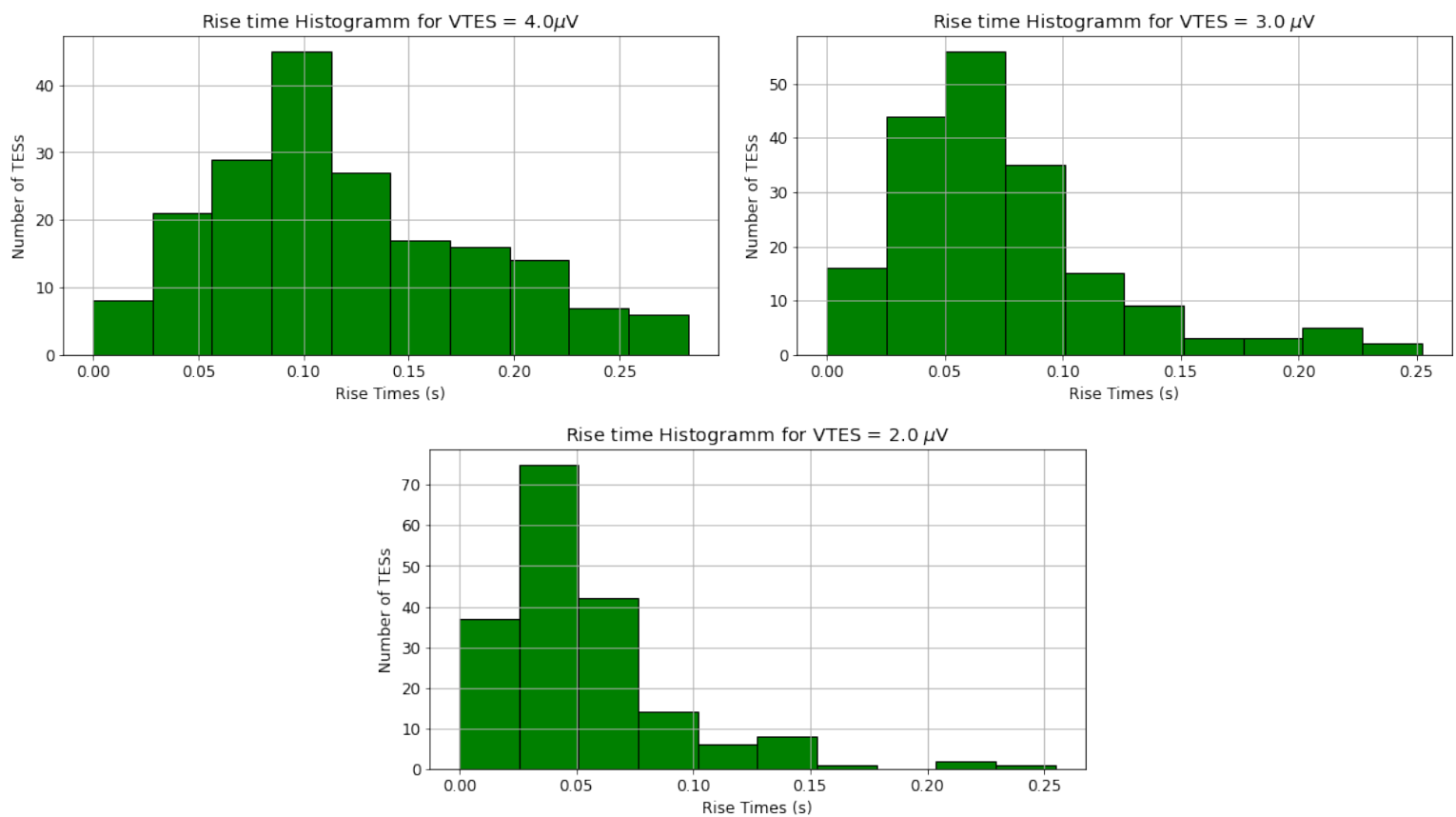

Figure 14. Distribution of the number of TES regarding the time constant and the TES voltage
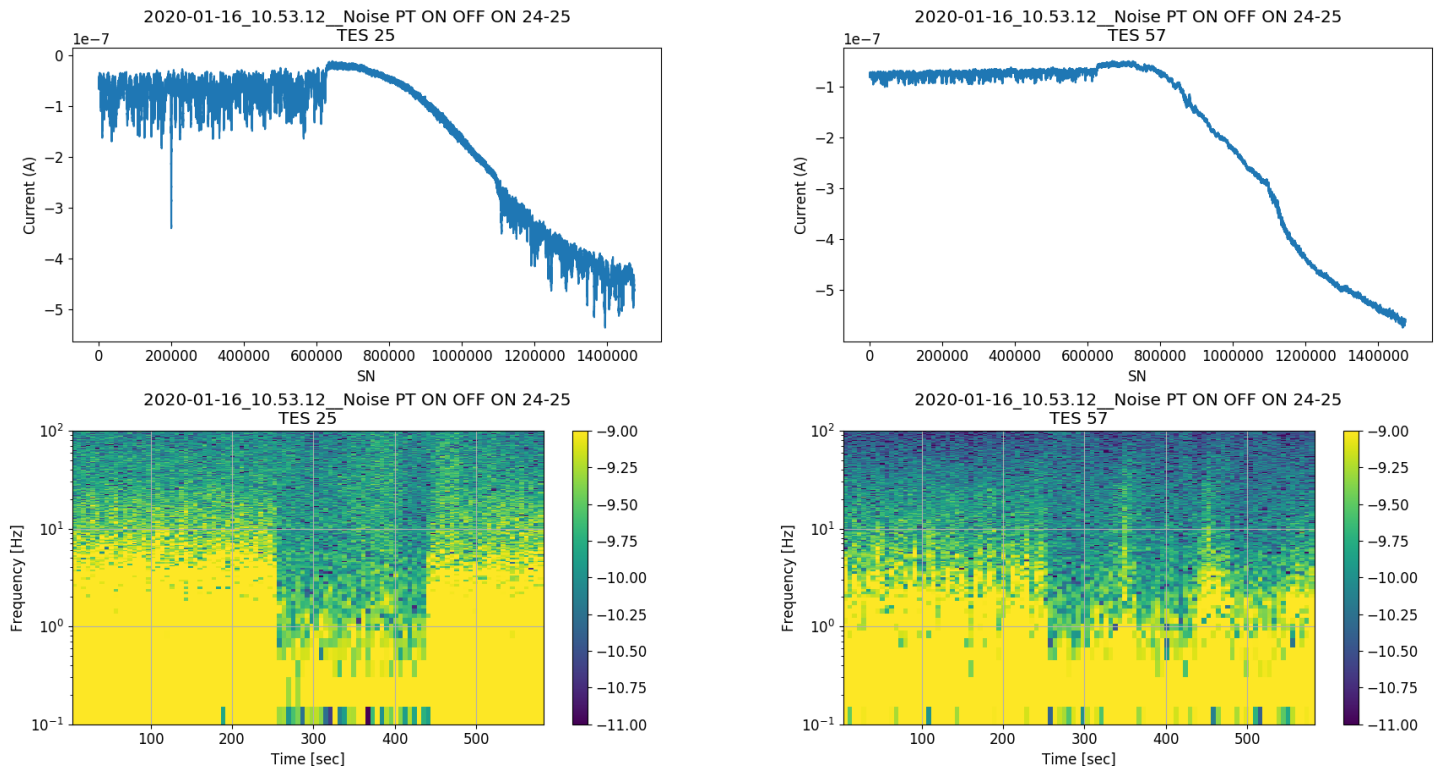

Figure 15. Examples of timeline and corresponding time-frequency analysis. The two pulse tubes are OFF for $\sim 240 \mathrm{~s}$ and $\sim 420$ s. (colorfull graph available online)

- We excited mechanically the cryostat with a speaker connected to an audio amplifier and a sine wave generator sweeping from $100 \mathrm{~Hz}$ to $1300 \mathrm{~Hz}$ in one hour. Figure 17 show timelines of TES and of the TES stage thermometer as a function of the excited frequency. Resonances are clearly seen, especially around $700 \mathrm{~Hz}$

To limit the impact of PT noise, we have coated the tubes connecting the compressors to the pulsing head with a neoprene coating. We have also isolated QUBIC from the ground to reduce the impact of low-frequency noise. 


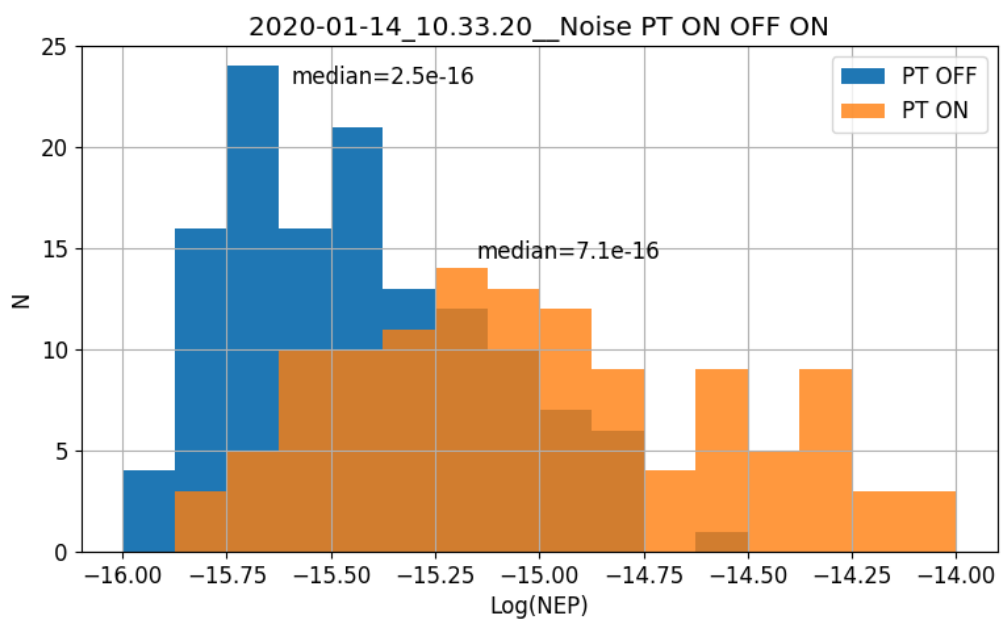

Figure 16. Histogram of NEP measured between $1 \mathrm{~Hz}$ and $2 \mathrm{~Hz}$ in the transition $\left(V_{\text {bias }}=1.5 \mathrm{~V}\right)$ with PTs ON and OFF. The response is assumed to be given by $1 / V_{T E S}$. The total number of TES are 130 and 120 respectively.(colorfull graph available online)

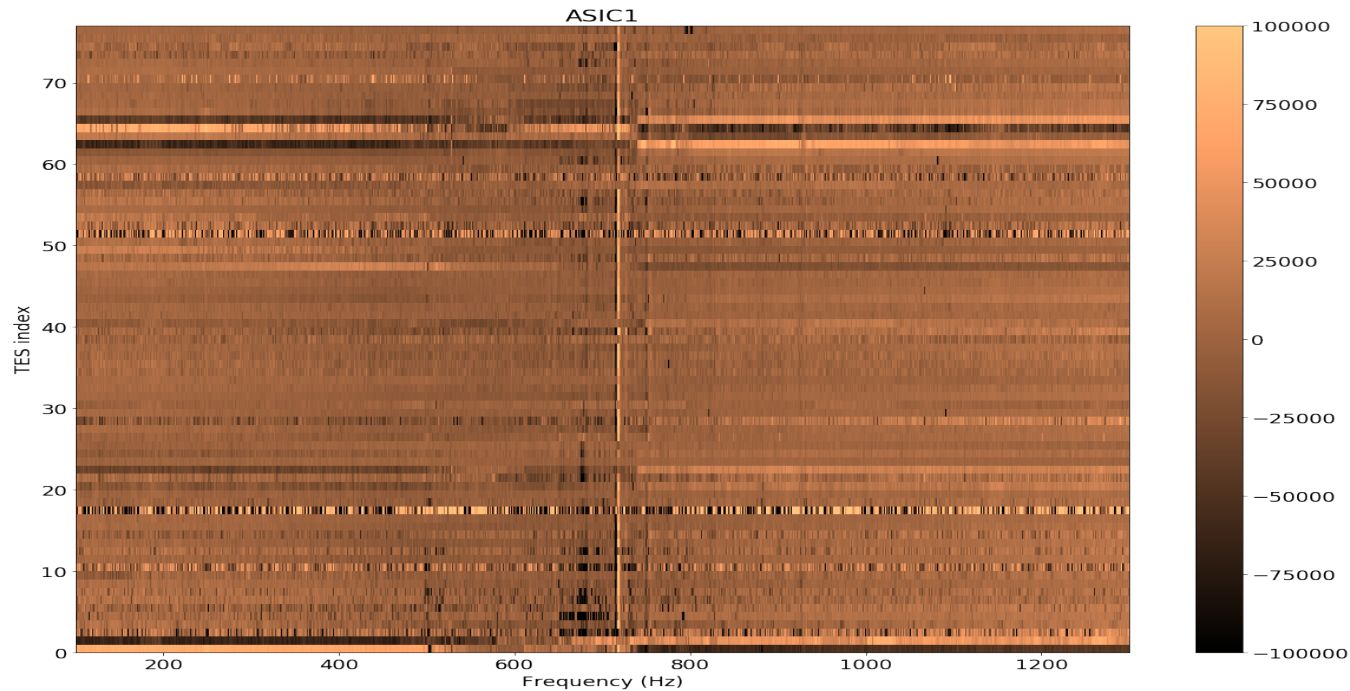

Figure 17. timelines in ADU of some TES as a function of the frequency of excitation.(colorfull graph available online)

\section{CONCLUSION}

The current underwent campain of QUBIC, shows very good results for the QUBIC detection chain. With a $79 \%$ yield of working TESs on the P87 matrix at 410mK and 90\% of working SQUIDs. This is the first experiment with a such amount of working TESs and SQUIDs together with a Time Constante between 100ms to 20ms.

\section{ACKNOWLEDGMENTS}

QUBIC is funded by the following agencies. France: ANR (Agence Nationale de la Recherche) 2012 and 2014, DIM-ACAV (Domaine dÕInteret MajeurÑAstronomie et Conditions dÕApparition de la Vie), CNRS/IN2P3 (Centre national de la recherche scientifique/Institut national de physique nuclaire et de physique des particules), CNRS/INSU (Centre national de la recherche scientifique/Institut national de sciences de lÕunivers), UnivEarthS Labex program at Sorbonne Paris Cité (ANR-10-LABX-0023 and ANR-11-IDEX-0005-02). Italy: CNR/PNRA (Consiglio Nazionale delle Ricerche/Programma Nazionale Ricerche in Antartide) until 2016, INFN (Istituto 
Nazionale di Fisica Nucleare) since 2017. Argentina: Secretara de Gobierno de Ciencia, Tecnologa e Innovacin Productiva, Comisin Nacional de Energa Atmica, Consejo Nacional de Investigaciones Cientficas y Tcnicas. UK: the University of Manchester team acknowledges the support of STFC (Science and Technology Facilities Council) grant ST/L000768/1. Ireland: James Murphy and David Burke acknowledge postgraduate scholarships from the Irish Research Council. Duc Hoang Thuong acknowledges the Vietnamese government for funding his scholarship at APC. Andrew May acknowledges the support of an STFC PhD Studentship.

\section{REFERENCES}

[1] Hamilton, J.-C., ..., Torchinsky, S., et al., "QUBIC - I: Overview and Science Program," ?, ? (Oct 2020). in preparation.

[2] Salatino, M., Bélier, B., Chapron, C., Hoang, D. T., Maestre, S., Marnieros, S., Marty, W., Montier, L., Piat, M., Prêle, D., Rambaud, D., Thermeau, J. P., Torchinsky, S. A., Henrot-Versillé, S., Voisin, F., Ade, P., Amico, G., Auguste, D., Aumont, J., Banfi, S., Barbarán, G., Battaglia, P., Battistelli, E., Baú, A., Bennett, D., Bergé, L., Bernard, J. P., Bersanelli, M., Bigot-Sazy, M. A., Bleurvacq, N., Bonaparte, J., Bonis, J., Bordier, G., Bréelle, E., Bunn, E., Burke, D., Buzi, D., Buzzelli, A., Cavaliere, F., Chanial, P., Charlassier, R., Columbro, F., Coppi, G., Coppolecchia, A., Couchot, F., D'Agostino, R., D'Alessand ro, G., de Bernardis, P., De Gasperis, G., De Leo, M., De Petris, M., Di Donato, A., Dumoulin, L., Etchegoyen, A., Fasciszewski, A., Franceschet, C., Gamboa Lerena, M. M., García, B., Garrido, X., Gaspard, M., Gault, A., Gayer, D., Gervasi, M., Giard, M., Giraud-Héraud, Y., Gómez Berisso, M., González, M., Gradziel, M., Grandsire, L., Guerrard, E., Hamilton, J. C., Harari, D., Haynes, V., Incardona, F., Jules, E., Kaplan, J., Korotkov, A., Kristukat, C., Lamagna, L., Loucatos, S., Louis, T., Lowitz, A., Lukovic, V., Luterstein, R., Maffei, B., Masi, S., Mattei, A., May, A. J., McCulloch, M. A., Medina, M. C., Mele, L., Melhuish, S., Mennella, A., Mundo, L. M., Murphy, J. A., Murphy, J. D., O'Sullivan, C., Olivieri, E., Paiella, A., Pajot, F., Passerini, A., Pastoriza, H., Pelosi, A., Perbost, C., Perdereau, O., Pezzotta, F., Piacentini, F., Piccirillo, L., Pisano, G., Polenta, G., Puddu, R., Ringegni, P., Romero, G. E., Schillaci, A., Scóccola, C. G., Scully, S., Spinelli, S., Stolpovskiy, M., Suarez, F., Tartari, A., Timbie, P., Tristram, M., Truongcanh, V., Tucker, C., Tucker, G., Vanneste, S., Viganò, D., Vittorio, N., Watson, B., Wicek, F., Zannoni, M., and Zullo, A., "Performance of NbSi transition-edge sensors readout with a 128 MUX factor for the QUBIC experiment," in [Millimeter, Submillimeter, and Far-Infrared Detectors and Instrumentation for Astronomy IX], Society of Photo-Optical Instrumentation Engineers (SPIE) Conference Series 10708, 1070845 (July 2018).

[3] Prêle, D., Voisin, F., Beillimaz, C., Chen, S., Piat, M., Goldwurm, A., and Laurent, P., "SiGe Integrated Circuit Developments for SQUID/TES Readout," Journal of Low Temperature Physics 193, 455-461 (Nov. 2018).

[4] Battistelli, E. S., Ade, P., Alberro, J. G., Almela, A., Amico, G., Arnaldi, L. H., Auguste, D., Aumont, J., Azzoni, S., Banfi, S., Battaglia, P., Baù, A., Bélier, B., Bennett, D., Bergé, L., Bernard, J. P., Bersanelli, M., Bigot-Sazy, M. A., Bleurvacq, N., Bonaparte, J., Bonis, J., Bottani, A., Bunn, E., Burke, D., Buzi, D., Buzzelli, A., Cavaliere, F., Chanial, P., Chapron, C., Charlassier, R., Columbro, F., Coppi, G., Coppolecchia, A., D'Alessandro, G., de Bernardis, P., De Gasperis, G., De Leo, M., De Petris, M., Dheilly, S., Di Donato, A., Dumoulin, L., Etchegoyen, A., Fasciszewski, A., Ferreyro, L. P., Fracchia, D., Franceschet, C., Lerena, M. M. G., Ganga, K., García, B., Redondo, M. E. G., Gaspard, M., Gault, A., Gayer, D., Gervasi, M., Giard, M., Gilles, V., Giraud-Heraud, Y., Berisso, M. G., González, M., Gradziel, M., Grandsire, L., Hamilton, J. C., Harari, D., Haynes, V., Henrot-Versillé, S., Hoang, D. T., Incardona, F., Jules, E., Kaplan, J., Korotkov, A., Kristukat, C., Lamagna, L., Loucatos, S., Louis, T., Luterstein, R., Maffei, B., Marnieros, S., Marty, W., Masi, S., Mattei, A., May, A., McCulloch, M., Medina, M. C., Mele, L., Melhuish, S., Mennella, A., Montier, L., Mousset, L., Mundo, L. M., Murphy, J. A., Murphy, J. D., Nati, F., Olivieri, E., Oriol, C., O’Sullivan, C., Paiella, A., Pajot, F., Passerini, A., Pastoriza, H., Pelosi, A., Perbost, C., Perciballi, M., Pezzotta, F., Piacentini, F., Piat, M., Piccirillo, L., Pisano, G., Platino, M., Polenta, G., Prêle, D., Puddu, R., Rambaud, D., Ringegni, P., Romero, G. E., Salatino, M., Salum, J. M., Schillaci, A., Scóccola, C., Scully, S., Spinelli, S., Stankowiak, G., Stolpovskiy, M., Suarez, F., Tartari, A., Thermeau, J. P., Timbie, P., Tomasi, M., Torchinsky, S., Tristram, M., Tucker, C., Tucker, G., Vanneste, S., Viganò, D., Vittorio, N., Voisin, F., Watson, B., Wicek, F., Zannoni, M., and Zullo, A., "QUBIC: The Q \& U Bolometric Interferometer for Cosmology," Journal of Low Temperature Physics 199, 482-490 (Feb. 2020). 